\title{
Gingival crevicular fluid and serum cystatin c levels in periodontal health and disease
}

\author{
Anuj Sharma ${ }^{\mathrm{a}}$, A.R. Pradeep ${ }^{\mathrm{a}, *}$, N.M. Raghavendra ${ }^{\mathrm{a}}$, P. Arjun ${ }^{\mathrm{b}}$ and Rahul Kathariya ${ }^{\mathrm{a}}$ \\ ${ }^{a}$ Department of Periodontics Bangalore Medical College, Government Dental College and Research Institute \\ Bangalore, Karnataka, India \\ ${ }^{\mathrm{b}}$ Bangalore Medical College, Bangalore, Karnataka, India
}

\begin{abstract}
Cystatin C (CSTC) is an inhibitor of cysteine proteinases and could play a protective and regulatory role under inflammatory conditions. The present study was designed to assess the concentration of CSTC in gingival crevicular fluid (GCF) and serum, to find out their association if any, in periodontal health and disease. 30 subjects were selected divided into 3 groups consisting of 10 subjects in each group based on clinical parameters: periodontally healthy group, gingivitis group and chronic periodontitis group, while, chronic periodontitis group after 8 weeks of the treatment (scaling and root planing) constituted after periodontal therapy group. GCF and serum samples were collected from all subjects to estimate the levels of CSTC by ELISA. The mean CSTC concentration in GCF and serum was observed to be the highest in periodontitis group and lowest in periodontally healthy group with intermediate concentration in gingivitis group and after periodontal therapy group. CSTC concentration in GCF and serum increased proportionally with the severity of periodontal disease (from health to periodontitis group) and decreased after treatment. This suggests that CSTC increases with disease progression to prevent further periodontal degeneration and decreases after treatment due to bone metabolic homeostasis. Further, longitudinal prospective studies involving larger population are needed to confirm the findings of present study and to better understand the role of CSTC in the pathogenesis of periodontal diseases.
\end{abstract}

Keywords: Cystatin C, gingival crevicular fluid, serum, chronic periodontitis, gingivitis

\section{Introduction}

Periodontitis is a disease of the periodontium characterized by irreversible loss of connective tissue attachment and supporting alveolar bone [1]. Destructive process in periodontitis is caused by an imbalance of the homeostasis between degradative enzymes such as the lysosomal cysteine proteinases, Cathepsins, and their inhibitors, Cystatins [2-4]. Cystatins are inhibitors of cysteine proteinases and could play a protective and regulatory role under inflammatory conditions.

Cystatin C (CSTC), also known as $\gamma$-trace (Gamma trace) or post $\gamma$ globulin proteinbelongs to family 2 of the Cystatin superfamily [5]. The synthesis of CSTC does not seem to be tissue-specific and all nucle-

*Corresponding author: Dr. Anuj Sharma, Dental Pearl, Lake View Apartment, Near Seva Sadan, Ranchi, Jharkhand 834001, India. Fax: +91 080 26703176; E-mail: dranuj31oct@yahoo.co.in. ated cells constitutively express and constantly secrete CSTC [6]. CSTC is considered as a marker of renal function in both healthy and diseased patients [7] and has been associated with various diseases with chronic inflammation and atherosclerosis [8], new onset hypertension [9], tumors [10], thyroid dysfunction [11] and Alzheimer's disease [12].

CSTC is believed to be the regulator of endogenous cysteine proteinase which are secreted or leaked from the lysosomes of dying or diseased cells [13]. Exogenous CSTC downregulates cathepsin K, one of the cysteine proteinases that play an important role in bone resorption [14]. Periodontal pathogens [Porphyromonas gingivalis, Actinobacillus actinomycetemcomitans (now known as Aggregatibacter actinomycetemcomitans)] and proinflammatory cytokines when added to gingival fibroblast induced by cyclosporine A, significantly increases the expression of CSTC compared with cyclosporine A alone [15]. Ska- 
leric et al. [16] reported that CSTC concentrations were significantly lower in gingival tissue samples from the sites with greater probing depth in subjects with inflamed gingival.

Decreased levels of CSTC were reported in gingival crevicular fluid (GCF) and total saliva in children with gingivitis compared to periodontally healthy children [17]. While Henskens et al. [18] found no CSTC levels in GCF samples while increased concentrations of CSTC were detected in whole saliva in both gingivitis and periodontitis subjects in comparison with healthy subjects. Other studies [19,20] from same group have reported the similar findings. Graziani F et al. [21,22] reported substantial reduction in serum CSTC concentration after non surgical periodontal therapy.

Considering the above studies, the levels of CSTC in GCF and saliva in periodontal diseases are controversial. Hence role of CSTC in periodontal disease is unclear and so it is difficult to understand that whether CSTC acts as a protective or destructive marker in the pathogenesis of periodontal disease. Thus, in view of the aforementioned findings, this study was undertaken to estimate the CSTC levels in GCF and serum from subjects with clinically healthy periodontium, gingivitis, chronic periodontitis and, subsequently, after initial periodontal therapy i.e. scaling and root planing (SRP) in the periodontitis subjects and to analyze the correlation between GCF and serum CSTC levels. After determining the levels of CSTC with severity of disease, it will be clear to correlate the role of CSTC with disease severity.

\section{Materials and methods}

\subsection{Study population and study groups}

The study population consisted of 30 age and gender matched subjects ( 15 women and 15 men; age range: 25-50 years) attending the outpatient clinic of the Department of Periodontics, Government Dental College and Research Institute, Bangalore, Karnataka, India. Ethical clearance was obtained from the Institutional Ethical Committee and Review Board. Written informed consent was obtained from those who agreed to participate voluntarily in the study. Inclusion criteria included subjects within the age group 25-50 years, who had not received periodontal therapy within the preceding 6 months and who had at least 20 natural teeth. Subjects with aggressive periodontitis, histo- ry of smoking, gross oral pathology, tumors or any other systemic diseases including bone diseases (such as rheumatoid and osteoarthritis) which can alter the course of periodontal disease, on any medication like bisphosphonates, cyclosporine-A, steroids, contraceptives and who had taken anti-inflammatory, antibiotics as well as pregnant and lactating females, were excluded from the study. The study was conducted from Feburary 2010 to May 2010.

Each subject underwent a full mouth periodontal probing and charting, along with the periapical radiographs using the long-cone technique [23]. The radiographic bone loss was recorded dichotomously (presence or absence) to differentiate chronic periodontitis patients from other groups. Furthermore, no delineation was attempted within the chronic periodontitis group based on the extent of alveolar bone loss.

Based on the gingival index (GI) [24], probing pocket depth (PPD), clinical attachment level (CAL) and radiographic evidence of bone loss, subjects were categorized into three groups. Periodontally healthy group consisted of 10 subjects with clinically healthy periodontium, with $\mathrm{GI}=0, \mathrm{PPD} \leqslant 3 \mathrm{~mm}, \mathrm{CAL}=0$ with no evidence of bone loss on radiographs. Gingivitis group consisted of 10 subjects who showed clinical signs of gingival inflammation with GI $>1, \mathrm{PPD} \leqslant 3 \mathrm{~mm}, \mathrm{CAL}$ $=0$ and no radiographic bone loss. Chronic periodontitis group consisted of 10 subjects who had signs of clinical inflammation with GI $>1$ along with a PPD $\geqslant$ $5 \mathrm{~mm}$ and $\mathrm{CAL} \geqslant 3 \mathrm{~mm}$ with the radiographic evidence of bone loss at more than 10 sites [25]. Patients were consecutively included into three groups according to the specific inclusion and exclusion criterias of each particular group. Patients with chronic periodontitis were treated with SRP, GCF and serum samples were collected 6-8 weeks after the treatment to constitute after-periodontal therapy group). A previously calibrated examiner (ARP) performed all the clinical assessments using a University of North Carolina-No.15 (UNC-15) periodontal probe. Calibration trials were performed prior to the study to ensure adequate intraexaminer reproducibility. No statistically significant difference was found between duplicate measurements ( $r=0.92$ for PPD and $r=0.91$ for CAL). The difference between the examinations was within $1 \mathrm{~mm}$ in $90 \%$ of PPD measurements and $92 \%$ of CAL measurements.

\subsection{GCF collection}

All the clinical and radiological examinations, group allocation and sampling site selection were performed 
by one examiner and the samples were collected on the subsequent day by a second examiner (AS). This was undertaken to prevent the contamination of GCF with blood associated with the probing of inflamed sites. Only one site per subject was selected as a sampling site in gingivitis and chronic periodontitis groups (groups 2 and 3), whereas, in the periodontally healthy group, to ensure the collection of an adequate amount of GCF, multiple sites with absence of inflammation were sampled. In gingivitis patients, the site with the highest clinical signs of inflammation (i.e. redness, bleeding on probing and edema), in the absence of clinical attachment loss, was selected. In chronic periodontitis patients, sites with $>2 \mathrm{~mm}$ clinical attachment loss were identified, and the site showing the highest clinical attachment loss and signs of inflammation, along with radiographic confirmation of bone loss, was selected for sampling.

After making the subjects sit comfortably in an upright position on the dental chair, the selected test site was air dried and isolated with cotton rolls. Without touching the marginal gingiva, supragingival plaque was removed to avoid contamination and blocking of the microcapillary pipette. GCF was collected by placing white color-coded $1-5 \mu$ l calibrated volumetric microcapillary pipettes (Sigma-Aldrich St. Louis, MO, USA) by placing the tip of the pipette extracrevicularly, until it gently touched the marginal gingiva, a standardized volume of $1 \mu \mathrm{l}$ unstimulated GCF was collected in 10 mins from each test site. The test sites which did not express standard volume $(1 \mu \mathrm{l})$ of GCF were excluded and micropipettes contaminated with blood and saliva was discarded. Periodontal treatment (SRP) was performed for both gingivitis and periodontitis patients at the same appointment after GCF collection. After 8 weeks, GCF was collected from same site of the subjects in chronic periodontitis group, and included in after periodontal therapy group. For this 8-week period, subjects were called at one-week interval and plaque control measures were performed.

The GCF collected was immediately sealed with sterile aluminium foil and transferred to plastic vial and stored at $-70^{\circ} \mathrm{C}$ till the time of the assay.

\subsection{Collection of serum}

Two milliliters of blood was collected from the antecubital fossa by venipuncture using a 20 -gauge needle with $2 \mathrm{ml}$ syringe and immediately transferred to the laboratory. The blood sample was allowed to clot at room temperature and, after $1 \mathrm{~h}$, serum was separated from blood by centrifuging at $3000 \mathrm{~g}$ for $5 \mathrm{~min}$. The extracted serum was immediately transferred to a plastic vial and stored at $-70^{\circ} \mathrm{C}$ till the time of assay.

\subsection{Determination of CSTC levels in samples}

CSTC levels from study participants were measured using a Human Cystatin C ELISA kit (BioVendorLaboratorni medicina, Modrice, Czech Republic Cata$\log$ No. RD191009100). Briefly, all reagents were allowed to warm to room temperature for at least $30 \mathrm{~min}$ prior to opening. Reagents were prepared according to the manufacturer's instructions immediately before use and mixed thoroughly during use without foaming. The GCF samples were expelled from the microcapillary pipettes with a jet of air using a blower provided with the pipettes and by further flushing them by a fixed amount of the diluent. After appropriate dilution, $100 \mu \mathrm{l}$ of diluted Standards, Quality Controls, Dilution Buffer (Blank) and samples, preferably in duplicates, were incubated (30 minutes at room temperature) in the wells pre-coated with antihuman CSTC antibody followed by washing of wells three times and horseradish peroxidase conjugated polyclonal antibodies against CSTC were added (incubated for $30 \mathrm{~min}-$ utes at room temperature). Finally tetramethylbenzidine substrate solution was added and reaction was stopped using stop solution ( $2 \mathrm{M}$ sulfuric acid) after 10 min. The absorbance was measured at $450 \mathrm{~nm}$ as primary wavelength and optical density values were obtained both for standards (provided with the kit) as well as samples. The concentration of CSTC in the tested samples was estimated using the reference calibrated standard curve, obtained by plotting the optical density values of the standards against their concentration.

\subsection{Statistical analysis}

Data were analyzed using a software program (SPSS version 10.5, SPSS Inc., Chicago, IL, USA). Based on the pilot study including five subjects in each group, the sample size was estimated at 10 subjects in each group to achieve power to detect a difference of 0.5 between the null hypotheses. Test for the validity of normality assumption using standardized range statistics was carried out and it was found that the assumption is valid. Accordingly parametric tests were carried out for comparing the means of CSTC concentration in different groups. Pair-wise comparison using scheff's test for GCF and serum CSTC was carried out to explore, which pair or pairs differ significantly at $5 \%$ level of 
Table 1

Descriptive statistics of the study population showing mean, standard deviation and range for the age, GI, CAL, PPD, GCF and serum CSTC concentrations, ANOVA test comparing the mean GCF and serum CSTC concentrations between four groups

\begin{tabular}{|c|c|c|c|c|c|}
\hline Parameters & $\begin{array}{l}\text { Periodontally healthy } \\
\text { group }(N=20) \\
\text { Mean } \pm \text { SD } \\
\text { Range (Min, Max.) }\end{array}$ & $\begin{array}{c}\text { Gingivitis } \\
\text { group }(N=20) \\
\text { Mean } \pm \text { SD } \\
\text { Range (Min, Max.) }\end{array}$ & $\begin{array}{l}\text { Chronic periodontitis } \\
\text { group }(N=20) \\
\text { Mean } \pm \mathrm{SD} \\
\text { Range (Min, Max.) }\end{array}$ & $\begin{array}{c}\text { After periodontal therapy } \\
\text { group }(N=20) \\
\text { Mean } \pm \text { SD } \\
\text { Range (Min, Max.) }\end{array}$ & F-value \\
\hline Age (Years) & $\begin{array}{c}28.7 \pm 3.6 \\
(25,36)\end{array}$ & $\begin{array}{c}28.5 \pm 3.1 \\
(25,35)\end{array}$ & $\begin{array}{c}32.6 \pm 7.5 \\
(25,47)\end{array}$ & $\begin{array}{c}32.6 \pm 7.5 \\
(25,47)\end{array}$ & \\
\hline GI & $0(-)$ & $\begin{array}{l}1.9 \pm 0.53 \\
(1.4,2.8)\end{array}$ & $\begin{array}{c}2.1 \pm 0.51 \\
(1.4,2.9)\end{array}$ & $\begin{array}{l}0.24 \pm 0.06 \\
(0.12,0.32)\end{array}$ & \\
\hline PPD (mm) & $\begin{array}{c}1.50 \pm 0.50 \\
(1,2)\end{array}$ & $\begin{array}{l}2.4 \pm 0.69 \\
(2,3)\end{array}$ & $\begin{array}{l}7.4 \pm 1.77 \\
(6,11)\end{array}$ & $\begin{array}{c}4.4 \pm 1.26 \\
(3,7)\end{array}$ & \\
\hline $\mathrm{CAL}(\mathrm{mm})$ & $0(-)$ & $0(-)$ & $\begin{array}{c}5.0 \pm 1.56 \\
(4,8)\end{array}$ & $\begin{array}{c}2.5 \pm 1.26 \\
(1,5)\end{array}$ & \\
\hline $\begin{array}{l}\text { GCF CSTC concentration } \\
(\mathrm{ng} / \mathrm{ml})\end{array}$ & $\begin{array}{c}2747.8 \pm 373.3 \\
(2257.8,3316.1)\end{array}$ & $\begin{array}{c}4632.8 \pm 347.7 \\
(4188.7,5158.3)\end{array}$ & $\begin{array}{c}6442.3 \pm 926.3 \\
(5168.0,7889.0)\end{array}$ & $\begin{array}{c}3534.5 \pm 527.6 \\
(2611.5,4309.4)\end{array}$ & $73.406^{*}$ \\
\hline $\begin{array}{l}\text { Serum CSTC concentration } \\
(\mathrm{ng} / \mathrm{ml})\end{array}$ & $\begin{array}{c}731.6 \pm 23.2 \\
(703.0,768.1)\end{array}$ & $\begin{array}{l}904.6 \pm 26.2 \\
(866.6,40.5)\end{array}$ & $\begin{array}{c}1204.5 \pm 30.9 \\
(1162.3,1253.9)\end{array}$ & $\begin{array}{c}829.0 \pm 26.8 \\
(788.5,867.1)\end{array}$ & $573.928^{*}$ \\
\hline
\end{tabular}

* Statistically significant at $p<0.05$.

significance. Paired t test was used to compare CSTC concentrations in GCF and serum in chronic periodontitis group and after periodontal therapy group. Pearson's correlation test was used to observe any correlation between the GCF and serum CSTC concentration and clinical parameters.

\section{Results}

The CSTC concentrations for all the groups are shown in Table 1. The mean CSTC concentration in GCF was observed to be the highest in chronic periodontitis group while least in periodontally healthy group. The mean CSTC concentration in GCF in gingivitis group and after periodontal therapy group was intermediate between above two groups. The mean CSTC concentration in serum was found to be least in periodontally healthy group, followed by after periodontal therapy group and gingivitis group, and highest in chronic periodontitis group. The F-value obtained for groups is 73.406 for GCF and 573.928 for serum $(p<0.001)$ as shown in Table 1 . Therefore the hypothesis of equality of means for concentration of CSTC in GCF and serum was rejected at $5 \%$ level of significance $(p<0.05)$, which indicate that the means differ significantly.

Further multiple comparisons using Scheff's test was carried out to find out which pair or pairs differ significantly. On comparison, between all the groups, the difference was statistically significant, both in GCF and serum $(p<0.05)$ (Table 2$)$. When chronic periodontitis group and after periodontal therapy group were compared using paired ' $t$ ' test, the difference in the con- centrations of CSTC was statistically significant in both GCF and serum $(p<0.05)$ (Table 2$)$.

Pearson's correlation coefficient test was done to find any correlation between the GCF and serum CSTC concentrations. The results showed a very strong and positive correlation in all the groups suggesting that GCF CSTC levels commensurate with that of serum and vice versa. GCF and serum CSTC levels showed strong positive correlation with clinical parameters in all groups $(p<0.05)$ except after periodontal therapy group (Table 3 ).

Differentiating values with probability 0.95 has shown that CSTC concentration in GCF and serum, $\leqslant 3937 \mathrm{ng} / \mathrm{ml}, 778 \mathrm{ng} / \mathrm{ml}$ respectively can be considered healthy, $3937 \mathrm{ng} / \mathrm{ml}-4590 \mathrm{ng} / \mathrm{ml}, 778 \mathrm{ng} / \mathrm{ml}-$ $1143 \mathrm{ng} / \mathrm{ml}$ for gingivitis and further increasing to $\geqslant$ $4590 \mathrm{ng} / \mathrm{ml}, 1143 \mathrm{ng} / \mathrm{ml}$ for chronic generalized periodontitis.

\section{Discussion}

The current study was carried out to explore the GCF and serum CSTC concentration with periodontal disease severity and results suggested that CSTC concentration increased from periodontal health to chronic periodontitis and decreases subsequently after non surgical periodontal therapy in both GCF and serum samples.

In the present study the influence of age and gender of the subjects on the CSTC concentration was minimised/nullified by including the equal number of males and female in each group and selecting the subjects within the specified age group of 25-50 years. 
Table 2

Pair-wise comparison using scheff's test for GCF and Serum CSTC among all the groups and Paired ' $t$ ' test to compare CSTC concentrations in GCF and serum in Chronic periodontitis group and after periodontal therapy group

\begin{tabular}{lrrrrrrr}
\hline & \multicolumn{3}{c}{ GCF } & \multicolumn{3}{c}{ Serum } \\
\cline { 2 - 3 } \cline { 6 - 8 } Study groups & $\begin{array}{c}\text { Mean } \\
\text { difference }\end{array}$ & Std. error & P value & & $\begin{array}{c}\text { Mean } \\
\text { difference }\end{array}$ & Std. error & P value \\
\hline Group PH and GG & -1885.02 & 264.26 & $<0.001^{*}$ & & -172.92 & 12.04 & $<0.001^{*}$ \\
Group PH and CP & -3694.52 & 264.26 & $<0.001^{*}$ & & -472.81 & 12.04 & $<0.001^{*}$ \\
Group PH and APT & 786.77 & 264.26 & $0.045^{*}$ & & -97.33 & 12.04 & $<0.001^{*}$ \\
Group GG and CP & -1809.49 & 264.26 & $<0.001^{*}$ & & -299.88 & 12.04 & $<0.001^{*}$ \\
Group GG and APT & 1098.25 & 264.26 & $0.003^{*}$ & & 75.59 & 12.04 & $<0.001^{*}$ \\
Group CP and APT & 2907.75 & 264.26 & $<0.001^{*}$ & & 375.47 & 12.04 & $<0.001^{*}$ \\
Group CP and APT & 2907.75 & & $<0.001^{*}$ & & 375.47 & & $<0.001^{*}$ \\
\hline
\end{tabular}

* Statistically significant at $p<0.05$

\#Paired ' $\mathrm{t}$ ' test.

PH- Periodontally Healthy Group;

GG- Gingivitis Group;

CP- Chronic periodontitis Group;

APT- After periodontal therapy Group.

Table 3

Pearson correlation coefficient test comparing GCF, serum CSTC with GI, PPD and CAL

\begin{tabular}{lcccccccc}
\hline Study & GCF Vs & \multicolumn{3}{c}{ GCF } & & \multicolumn{3}{c}{ serum } \\
\cline { 3 - 5 } \cline { 6 - 8 } Groups & Serum & GI & PPD & CAL & & GI & PPD & CAL \\
\hline PH & $0.996^{*}$ & $-0.871^{*}$ & - & - & & $0.864^{*}$ & - & \\
GG & $0.993^{*}$ & $0.918^{*}$ & $0.891^{*}$ & $-0.935^{*}$ & & $0.923^{*}$ & - & \\
CP & $0.988^{*}$ & $0.994^{*}$ & $0.939^{*}$ & $0.937^{*}$ & & $0.984^{*}$ & $0.902^{*}$ & $0.899^{*}$ \\
APT & $0.992^{*}$ & 0.962 & 0.439 & 0.347 & & 0.063 & 0.490 & 0.389 \\
\hline * Statistically significant at $p<0.05$. & & & & & \\
PH- Periodontally Healthy Group; & & & & & & \\
GG- Gingivitis Group; & & & & & &
\end{tabular}

In the present study, the extracrevicular (unstimulated) method of GCF collection using microcapillary pipettes was employed to ensure atraumatism, to obtain an undiluted sample of native GCF whose volume can be accurately assessed and also to avoid nonspecific attachment of the analyte to filter paper fibers [26]. The disadvantage of this method is the possibility of trauma to the marginal gingiva, and care was taken to avoid this during GCF collection. Furthermore, loss of GCF due to sticking of the sample to the capillary walls was avoided by flushing the capillary with a fixed amount of diluent, which was taken into account during the final calculations. In the present study, the use of commercially available, sensitive ELISA kit to quatify CSTC from selected sites allowed us to avoid pooling of GCF samples from multiple sites or subjects.

Our study comprised of four groups (healthy, gingivitis, chronic periodontitis and chronic periodontitis after treatment) in addition to the previous study by ulker et al. [17] where the CSTC levels were only investigated in periodontally healthy children and chil- dren with chronic gingivitis. The additional groups of periodontitis and after treatment in our study helped us to evaluate the role of CSTC in different stages of periodontal disease and the effect of periodontal therapy on CSTC concentrations which can further confirm its role in periodontal disease.

The results of the present study indicated that concentration of CSTC in GCF increased progressively from healthy to periodontitis sites, while in gingivitis the mean concentration of CSTC fell between the above two groups. The results of our study are contrary to Ulker et al. [17] who reported decreased levels of CSTC in GCF and total saliva in patients with gingivitis compared to periodontally healthy children and Henskens et al. [18] who has not found CSTC in GCF samples. Use of filter paper strip for GCF collection and immunoblotting technique used for detection of CSTC in GCF in Henskens et al. [18] study can be the reason for no expression of CSTC in GCF samples which has been overcome by using microcapillary pipettes for GCF collection and sensitive ELISA kits in the cur- 
rent study. In the present study increased CSTC level in GCF indicates amplification of osteoclastic activity signals and so CSTC increase for counterbalance these pathways in the inflamed periodontium as in gingivitis and periodontitis.

The mean CSTC levels in serum increased progressively from health to gingivitis and periodontitis. Further, when the concentration of CSTC in GCF was compared to that of serum, the concentration of CSTC in GCF was significantly higher than corresponding serum concentration in all the groups. Possible reason can be the inflammatory cytokines associated with periodontitis which stimulate the production of lysosomal cathepsins, and increased plasma concentrations of cystatin C, a cathepsin inhibitor, may reflect, at least in part, an attempt to counterbalance a potentially damaging increased osteoclastic activity. Increase in serum concentration of CSTC has been detected in various other chronic inflammatory diseases $[8,9,11,12]$. Leung et al. [8] reported that high CSTC concentrations are directly related to both inflammation and atherosclerosis and involved in the pathogenesis of atherosclerosis. Kestenbaum et al. [9] evaluated that for each $15 \mathrm{nmol} / \mathrm{L}$ $(0.2 \mathrm{mg} / \mathrm{L})$ increase in CSTC concentration there was a $15 \%$ greater incidence of hypertension. So elevated serum CSTC levels due to periodontal disease can increase the risk of cardiac diseases.

Previous studies [21,22] reported reduction in serum CSTC levels after non surgical periodontal therapy but they considered that the inflammatory markers from periodontal disease may trigger a systemic acute-phase systemic inflammatory response, characterized by increased levels of acute phase proteins which affect renal function. Non surgical periodontal therapy of such patients can lead to reduction of systemic inflammation and improve renal functionality assessed by CSTC (Measuring GFR) [21,22]. While current study indicates that production of CSTC increases in local periodontal tissue as a protective marker and spillover from local tissue to serum leads to elevation of serum CSTC in transformation from periodontal health to disease, and after non surgical periodontal therapy, local inflammatory component subsided, reduction in CSTC levels takes place in periodontal tissues and so in serum.

The variability of CSTC concentration within the patients of each group can be attributed to the different stages of disease process at the time of collection of GCF and serum samples. The few samples of gingivitis group showed values nearing that of chronic periodontitis group especially in GCF, which could be attributed to near conversion of gingivitis lesions to chronic periodontitis lesions that is not clinically detectable. A few of the samples of periodontally healthy group showed values nearing those of gingivitis group which could to because of subclinical levels of inflammation in the clinically healthy tissues. Further there was significant overlap between gingivitis group and the after periodontal therapy group, which can be due to the individual variation in the resolution of periodontitis after treatment.

It has been proposed that peptidyldiazomethane, mimicking part of the proposed substrate like-binding region of CSTC, displays irreversible inhibition of papain and a Streptococcal cysteine proteinase and appears to have a potential as an antimicrobial drug [27]. In addition, recombinant human CSTC inhibits cysteine proteinases papain with same efficiency of natural CSTC [28]. Thus in future CSTC and recombinant CSTC may have important implications in the design of novel therapies for periodontal disease.

In summary, CSTC concentration was found to be elevated from periodontally healthy group to chronic periodontitis group in both GCF and serum samples and decrease in after periodontal therapy group. This suggests that CSTC acts as anti-inflammatory marker in GCF and serum.

\section{Conclusion}

CSTC concentration in GCF and serum increases proportionally with the severity of periodontal disease. Further, the treatment aimed at arresting periodontal disease, resulted in statistically significant reduction in the levels of CSTC both in GCF and serum proportionally. This suggests that CSTC increases with disease progression to prevent further periodontal degeneration and decreases after treatment due to bone metabolic homeostasis. The role of CSTC as an "inhibitory biomarker of osteoclastic activity" can be explored in future as a potential therapeutic target in the treatment of periodontal disease. Further, longitudinal prospective studies involving larger population are needed to confirm the findings of present study and to better understand the role of CSTC in the pathogenesis of periodontal diseases.

\section{Acknowledgments}

The authors express their thanks to Mr. Jagannatha P S, a Statistician, Rajajinagar, Bangalore for carrying all required statistics. The authors report no conflicts of interest related to this study. 


\section{References}

[1] B.L. Pihlstrom, B.S. Michalowicz and N.W. Johnson, Periodontal diseases, Lancet 366 (2005), 1809-1820.

[2] M. Abrahamson, M. Wikstrom, J. Potempa, S. Renvert and A. Hall, Modification of cystatin C activity by bacterial proteinases and neutrophil elastase in periodontitis, Mol Pathol 50 (1997), 291-297,

[3] M.F. Blankenvoorde, Y.M. Henskens, G.A. van der Weijden, P.A. van den Keijbus, E.C. Veerman and A.V. Nieuw Amerongen, Cystatin A in gingival crevicular fluid of periodontal patients, J Periodontal Res 32 (1997), 583-588.

[4] M. Mogi and J. Otogoto, Expression of cathepsin-K in gingival crevicular fluid of patients with periodontitis, Arch Oral Biol 59 (2007), 894-898.

[5] M. Abrahamson, G. Salvesen, A.J. Barrett and A. Grubb, Isolation of six cysteine proteinase inhibitors from human urine. Their physicochemical and enzyme kinetic properties and concentrations in biological fluids, J Biol Chem 261 (1986), 11282-11289.

[6] D.J. Newman and Cystatin C, Ann Clin Biochem 39 (2002), 89-104.

[7] A. Tanaka, K. Suemaru and H. Araki, A new approach for evaluating renal function and its practical application, Journal of Pharmacological Sciences 105 (2007), 1-5.

[8] J. Leung-Tack, C. Tavera, M.C. Gensac, J. Martinez and A. Colle, Modulation of phagocytosis-associated respiratory burst by human cystatin C: role of the N-terminal tetrapeptide Lys-Pro-Pro-Arg, Exp Cell Res 188 (1990), 16-22.

[9] B. Kestenbaum, K.D. Rudser, B.I. De et al., Differences in kidney function and incident hypertension: the multi-ethnic study of atherosclerosis, Ann Intern Med 148 (2008), 501-508.

[10] P. Strojan, I. Oblak, B. Svetic, L. Smid and J. Kos, Cysteine proteinase inhibitor cystatin $\mathrm{C}$ in squamous cell carcinoma of the head and neck: relation to prognosis, British Journal of Cancer 90 (2004), 1961-1968.

[11] V. Jayagopal, B.G. Keevil, S.L. Atkin, P.E. Jennings and E.S. Kilpatrick, Paradoxical changes in cystatin $\mathrm{C}$ and serum creatinine in patients with hypo- and hyperthyroidism, Clin Chem 49 (2003), 680-681.

[12] E. Levy, M. Sastre, A. Kumar et al., Codeposition of cystatin $\mathrm{C}$ with amyloid- $\beta$ protein in the brain of Alzheimer disease patients, J Neuropathol Exp Neurol 60 (2001), 94-104.

[13] A. Grubb and H. Loftberg, Human gamma-trace. Structure and clinical use of concentration measurements, Scand J Clin Lab Invest 45 (1985), 7-13.

[14] C. Schick, P.A. Pemberton, G.P. Shi et al., Cross-class inhibition of the cysteine proteinases chatepsins $\mathrm{K}, \mathrm{L}$, and $\mathrm{S}$ by the serpin squamous cell carcinoma antigen. 1: A kinetic analysis, Biochem 37 (1998), 5258-5266.
[15] C.H. Tsai, S.F. Yang, F.M. Huang and Y.C. Chang, The upregulation of cystatin $\mathrm{C}$ in human gingival fibroblasts stimulated with cyclosporine A, J Periodont Res 44 (2009), 459-464.

[16] U. Skaleric, J. Babnik, V. Curin, T. Lah and V. Turk, Immunochemical Quantitation of Cysteine Proteinase Inhibitor Cystatin $\mathrm{C}$ in inflamed human gingiva, Archives of Oral Biology 34 (1989), 301-305.

[17] A.E. Ulker, O. Tulunoglu, N. Ozmeric, M. Can and S. Demirtus, The Evaluation of Cystatin C, IL- $1 \beta$, and TNF- $\alpha$ Levels in Total Saliva and Gingival Crevicular Fluid From 11- to 16-Year-Old Children, J Periodontol 79 (2008), 854-860.

[18] Y.M.C. Henskens, E.C.I. Veerman, M.S. Mantel, U.V. Velden and A.V.N. Amerongen, Cystatin S and C in Human Whole Saliva and in Glandular Saliva in Periodontal Health and Disease, J Dent Res 73 (1994), 1606-1614.

[19] Y.M.C. Henskens, U. Van der Velden, E.C. Veerman and A.V. Nieuw Amerongen, Protein, albumin and cystatin concentrations in saliva of healthy subjects and of patients with gingivitis or periodontitis, J Periodontal Res 28 (1993), 43-48.

[20] Y.M.C. Henskens, P.A. Van den Keijbus, E.C. Veerman et al., Protein composition of whole and parotid saliva in healthy and periodontitis subjects. Determination of cystatins, albumin, amylase and IgA, J Periodontal Res 31 (1996), 57-65.

[21] F. Graziani, S. Cei, M. Tonetti et al., Systemic inflammation following non-surgical and surgical periodontal therapy, J Clin Periodontol 37 (2010), 848-854.

[22] F. Graziani, S. Cei, F. La Ferla, M. Vano, M. Gabriele and M. Tonetti, Effects of non-surgical periodontal therapy on glomerular filtration rate of the kidney: an exploratory trial, $J$ Clin Periodontol 37 (2010), 638-643.

[23] A. Khocht, M. Janal, L. Harasty and K. Minchang, Comparison of direct digital and conventional intraoral radiographs in detecting alveolar bone loss, JADA 134 (2003), 1468-1475.

[24] H. Loe and J. Silness, Periodontal disease in pregnancy. I prevalence and severity, Acta Odontol Scand 21 (1963), 533.

[25] D.F. Kinane and J. Lindhe, Chronic periodontitis, in: Clinical Periodontology and Implant Dentistry, J. Lindhe, T. Karring and N.P. Lang, eds, Oxford: Blackwell, 2003, pp. 209-215.

[26] T. Sueda, J. Bang and G. Cimasoni, Collection of gingival fluid for quantitative analysis, J Dent Res 48 (1969), 159.

[27] L. Bjorck, P. Akesson, M. Bohus et al., Bacterial growth blocked by a synthetic peptide based on the structure of a human proteinase inhibitor, Nature 337 (1989), 385-338.

[28] M. Abrahamson, R.W. Mason, H. Hansson, D.J. Buttle, A. Grubb, K. Ohlsson and C. Human cystatin, Role of the Nterminal segment in the inhibition of human cysteine proteinases and in its inactivation by leucocyte elastase, Biochem J 273 (1991), 621-626. 


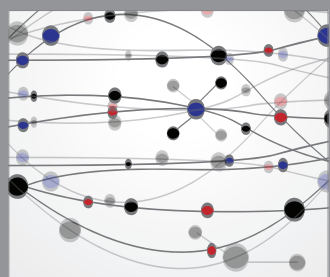

The Scientific World Journal
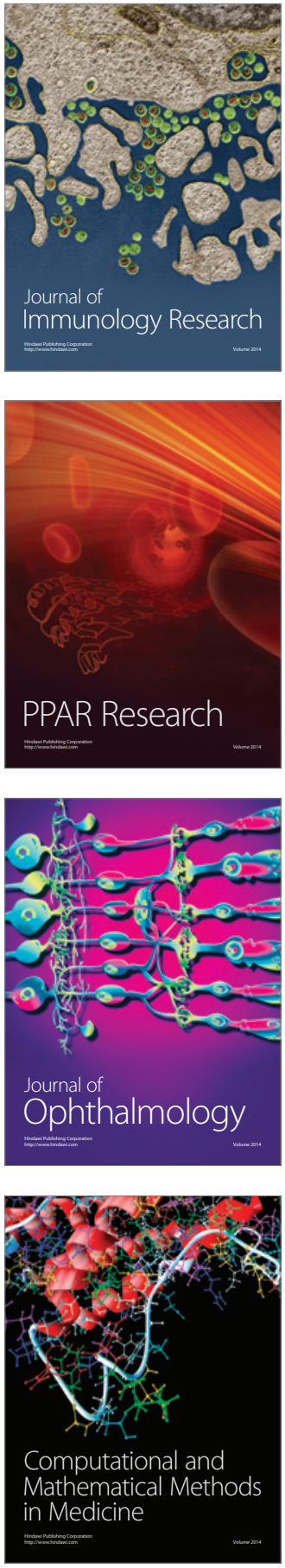

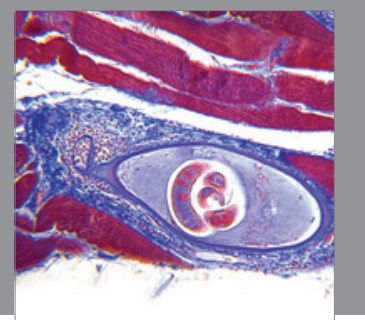

Gastroenterology

Research and Practice
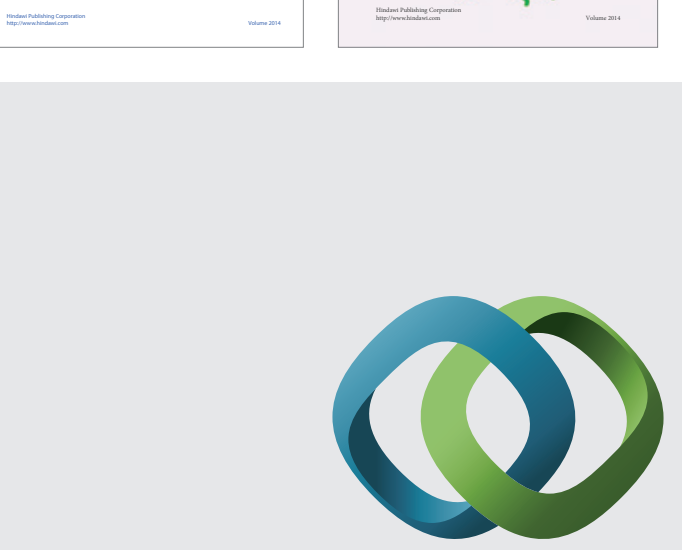

\section{Hindawi}

Submit your manuscripts at

http://www.hindawi.com
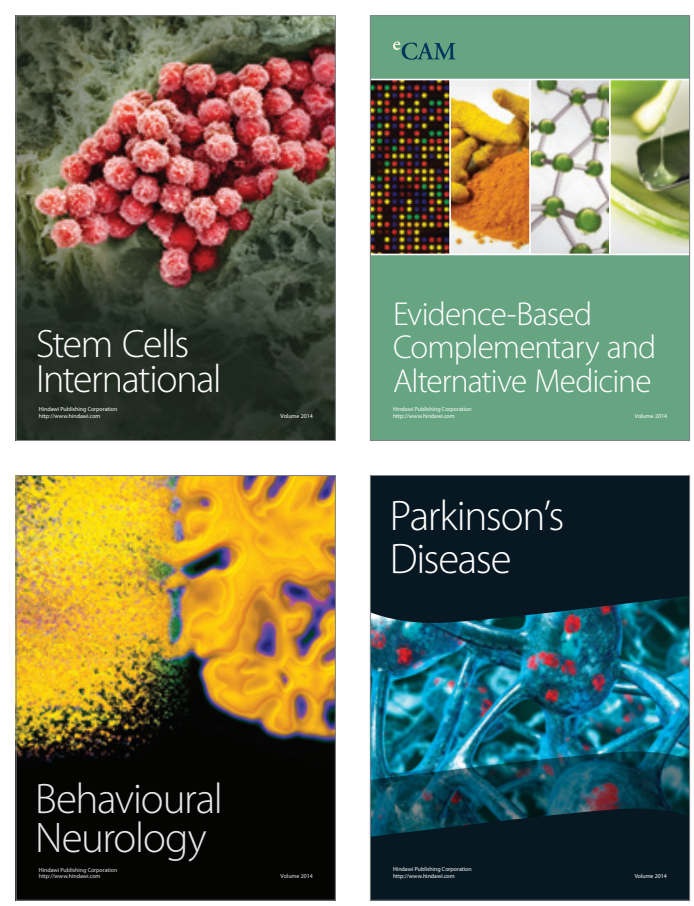

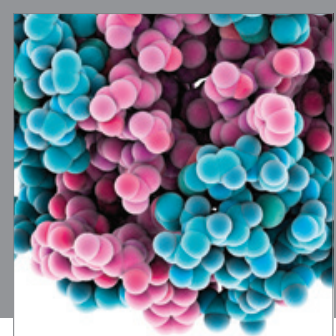

Journal of
Diabetes Research

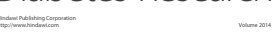

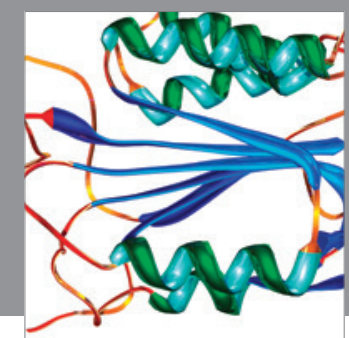

Disease Markers
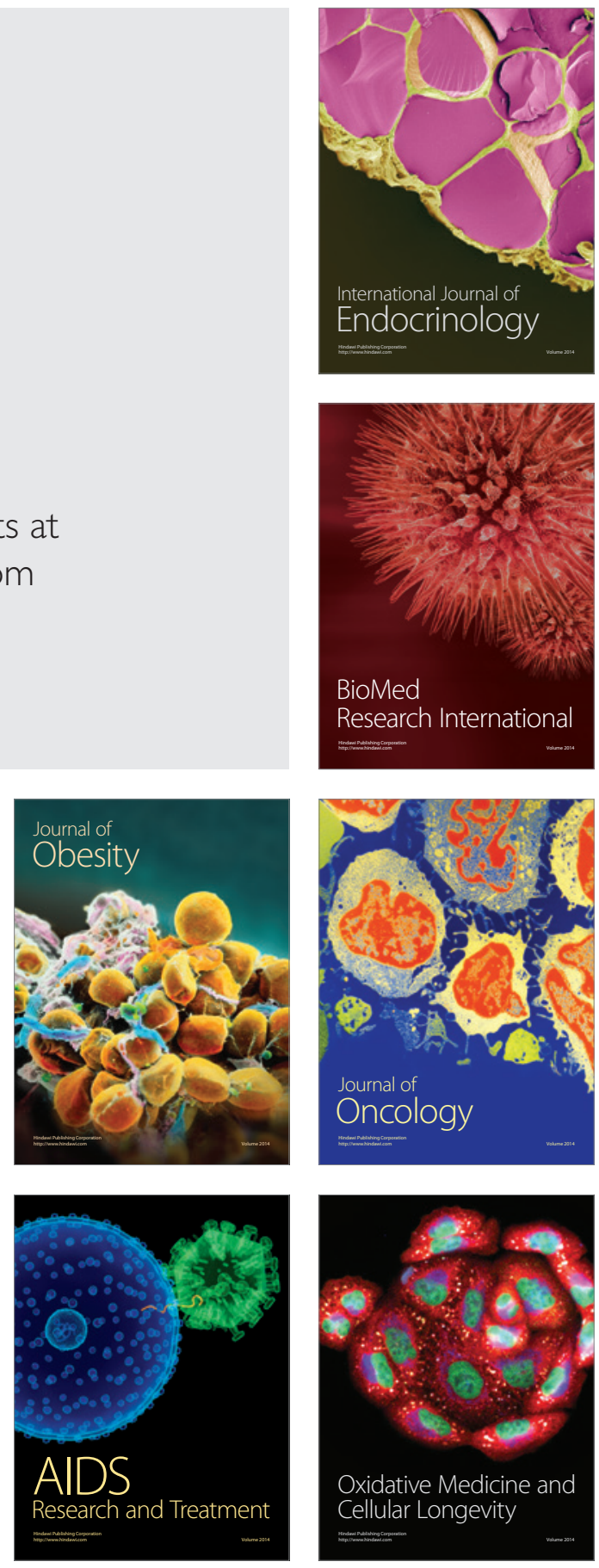